\section{Los atributos de un tutor clínico de excelencia: una revisión sistemática}

\author{
JONATHAN RODRÍGUEZ-CABELLO ${ }^{1, a}$, NICOLÁS ORTIZ-LÓPEZ $Z^{1, a}$, \\ CAROLINA OLEA-GANGAS ${ }^{1, a}$, FELIPE CORTÉS-CHAU ${ }^{1, a}$, \\ ÓSCAR JEREZ YÁÑEZ ${ }^{2, \mathrm{~b}}$
}

\section{A systematic review about the most important attributes of clinical teachers}

\begin{abstract}
Knowing what characterizes an excellent teacher is relevant to guide training, evaluation, and continuous improvement of the clinical teacher. We performed a systematic review using the PRISMA protocol, aiming to identify the attributes of an excellent clinical teacher. MEDLINE, ERIC, ScienceDirect, and Scopus databases were searched for articles published in English and Spanish, between 2007 and 2019. Two independent reviewers extracted and synthesized data from articles that met the PRISMA pre-established criteria. Twelve studies met the inclusion criteria. Of the 106 attributes reported by the studies, 49 (46,2\%) were generic. The most frequently mentioned attribute was respectfulness. Forty-six attributes (43,3\%) were pedagogical. Feedback and planning were those most frequently mentioned. Eleven attributes $(10,3 \%)$ were disciplinary and clinical skills was the most mentioned. We conclude that generic and pedagogical dimensions had a greater representation in the literature, in comparison with the disciplinary dimension. This could be explained, considering that having adequate clinical skills is the minimal necessary requisite to become a clinical teacher, but does not make a difference in how their performance is perceived, contrary to the generic and pedagogical dimensions.
\end{abstract}

(Rev Med Chile 2020; 148: 1339-1349)

Key words: Education, Medical; Mentoring; Preceptorship; Teaching.
${ }^{1}$ Centro de Enseñanza y

Aprendizaje, Facultad de

Medicina, Universidad de Chile.

Santiago, Chile.

${ }^{2}$ Departamento de Educación

Ciencias de la Salud, Facultad de

Medicina, Universidad de Chile.

Santiago, Chile.

aInterno(a) de Medicina.

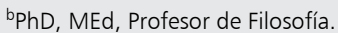

Trabajo no recibió financiamiento. Los autores declaran no tener conflictos de interés.

Recibido el 30 de marzo de 2019 aceptado el 3 de julio de 2020.

Correspondencia a: Jonathan Rodríguez Cabello Avenida Parque Norte 7131, Renca. Santiago, Chile. jonathan.rodriguez@ug.uchile.cl

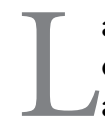

a adquisición de conocimientos, habilidades clínicas y formación ética es una piedra angular en la educación médica a cualquier nivel, por lo que la presencia de un tutor clínico o una tutora clínica de excelencia es un elemento clave para lograr este objetivo ${ }^{1,2}$. Un tutor clínico corresponde a un médico que supervisa a sus estudiantes durante su práctica clínica ${ }^{3}$. Identificar cuáles son los atributos de un tutor clínico de excelencia es esencial para desarrollar diferentes herramientas y experiencias que permitan mejorar la formación médica, así como también para la creación de programas de formación de tutores clínicos.

En relación a la temática, se han publicado dos revisiones sistemáticas en los últimos años. El primero sobre las cualidades de un buen tutor clínico ${ }^{2}$ y el segundo sobre los atributos que hacen del tutor clínico un modelo a seguir ${ }^{4}$. Estas revisiones destacan características como la paciencia, integridad, invertir tiempo en la relación médico-paciente, habilidades comunicacionales, mantener una buena relación con los estudiantes y un buen ambiente de aprendizaje, el conocimiento clínico, el razonamiento clínico y el entusiasmo, entre otras cualidades $^{2,4}$. El presente artículo contribuye en la actualización desde los últimos hallazgos en esta área, utilizando un protocolo sistemático y una síntesis de los resultados aglutinados en dimensiones. En este sentido, el objetivo de este estudio 
es brindar evidencia y respuesta a la pregunta: ¿Cuáles son los atributos de un tutor clínico de excelencia? Así como clasificar estos atributos en dimensiones disciplinaria, pedagógica y genérica.

\section{Método}

\section{Protocolo de revisión}

Esta revisión se elaboró siguiendo las recomendaciones propuestas en la declaración Preferred Reporting Items for Systematic Reviews and Meta-Analyses (PRISMA), descritas en el documento de explicación y elaboración de PRISMA ${ }^{5}$. Se utilizó el protocolo mencionado por ser un estándar en la realización de revisiones sistemáticas en la literatura biomédica en la actualidad. No se requirió aprobación ética para este estudio, ya que corresponde a una revisión sistemática de la literatura publicada. La calidad de los estudios incluidos fue garantizada con la selección exclusiva de artículos publicados en revistas revisadas por pares.

\section{Estrategia de búsqueda}

Se realizó una búsqueda sistemática en cuatro bases de datos (MEDLINE, ERIC, ScienceDirect y Scopus) para artículos publicados en inglés o español, en revistas revisadas por pares, entre enero de 2007 y diciembre de 2019. Los términos de búsqueda incluyeron los ámbitos de entrenador clínico, la efectividad o calidad del maestro y la educación médica. Se utilizó una estrategia de búsqueda de palabras clave y encabezados de temas médicos (MeSH) para PubMed. La estrategia de búsqueda de MeSH incluyó los términos "Medical, Education", y "Schools, Medical". Se utilizó una estrategia de búsqueda de palabras clave y tesauros para ERIC; que incluyó los términos "Teacher Effectiveness", "Teacher skills", "Medical School Faculty" "Preceptors (Medicine)", "Medical Education" " "Medical School”. La búsqueda en las bases de datos restantes se efectuó utilizando vocabularios controlados (Tabla 1). Adicionalmente, se realizó una búsqueda manual de la literatura basados en las referencias de los artículos seleccionados y se exploró bibliografía adicional en cuatro revistas de educación médica (Academic Medicine, Journal of Postgraduate Medicine, Medical Education, Medical Teacher) publicadas dentro del año anterior a la búsqueda.

\section{Proceso de selección}

Dos de los autores (C.O., J.R.) revisaron de forma independiente los títulos y resúmenes de publicaciones identificados en la búsqueda. Posteriormente se seleccionaron los artículos relevantes para su posible inclusión (Tabla 1). Las discrepancias fueron resueltas mediante discusión o la participación de un tercer revisor (N.O.L.). El texto completo de estos artículos fue recuperado para una revisión adicional (Figura 1).

Se incluyeron estudios originales, tanto cualitativos como cuantitativos, que describen las características del tutor clínico de excelencia para estudiantes de escuelas de medicina de pregrado y posgrado. Se excluyeron artículos centrados en áreas no médicas, residentes como tutores clínicos, aprendizaje asistido por pares, programas de capacitación para tutores clínicos, revisiones, editoriales y comentarios.

\section{Proceso de recopilación de datos y elementos de datos}

Dos revisores extrajeron de forma independiente todos los datos mediante el formulario de extracción de datos estandarizado. Resolvimos discrepancias por discusión. Los campos de extracción de datos incluyeron autores, año de publicación, diseño del estudio, participantes, medidas de resultado y resultados. La discusión con un tercer autor (N.O.L.) resolvió cualquier diferencia en la extracción de datos.

\section{Clasificación de habilidades}

Cuatro revisores revisaron las habilidades relevantes mencionadas en los artículos, para posteriormente agruparlas en atributos. Cada habilidad se clasificó en una de las siguientes dimensiones: genérica, pedagógica y disciplinaria. Además, para definir un atributo como significativo se propuso un umbral de $25 \%$ de apariciones en la literatura seleccionada, es decir, el atributo debe haber sido mencionado en al menos 3 de los 12 artículos para ser considerado significativo.

\section{Resultados}

\section{Selección de los estudios}

La revisión sistemática identificó un total de 897 artículos (416 estudios de Science Direct, 209 de Medline, 191 de Scopus y 81 de ERIC). De es- 
Tabla 1. Criterios de inclusión y exclusión aplicados en la búsqueda bibliográfica

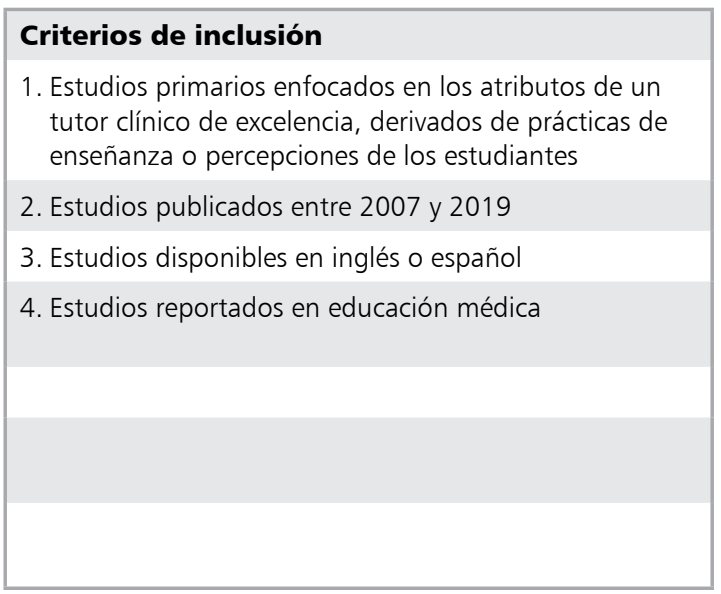

\section{Criterios de exclusión}

1. Estudios secundarios, como revisiones, editoriales, comentarios y libros

2. Estudios publicados antes de 2007

3. Estudios publicados en idiomas distintos al inglés o español

4. Estudios enfocado en la validación o construcción de instrumentos

5. Estudio de pares o por residentes médicos

6. Estudios enfocados en la efectividad de programas de entrenamiento de tutores clínicos

7. Estudios reportados en carreras de la salud distintas a la medicina

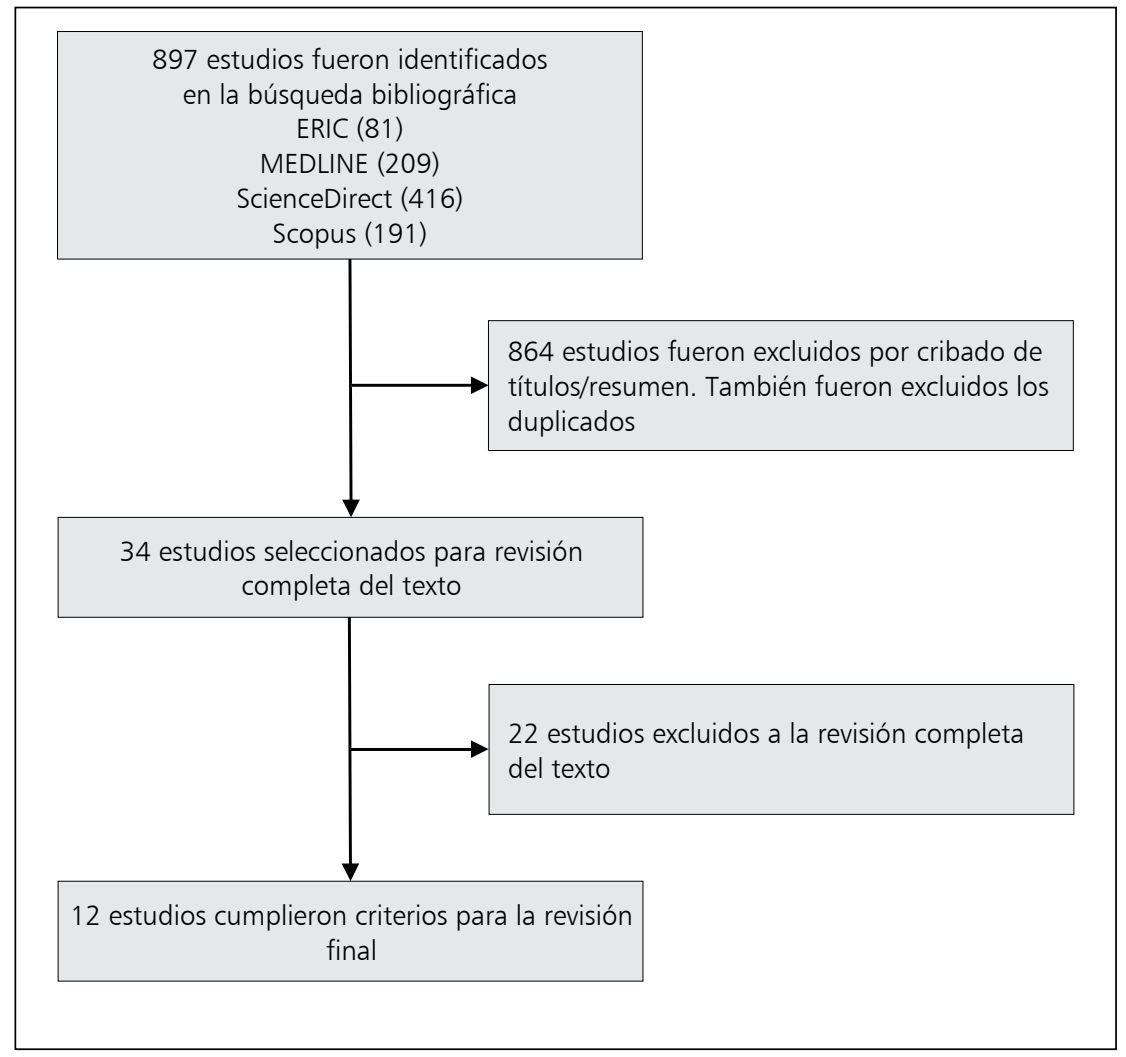

Figura 1. Diagrama de flujo de la búsqueda en la literatura y proceso de selección de estudios en una revisión sistemática de la literatura sobre atributos de un tutor clínico de excelencia. Artículos publicados entre enero de 2007 y diciembre de 2019.

tos, se excluyeron 864 artículos. De los artículos restantes, 34 fueron seleccionados en función de su título y resumen. Se realizó una revisión de texto completo en los artículos seleccionados.
Veintidós estudios no cumplieron los criterios de inclusión. Finalmente, 12 estudios cumplieron con todos los criterios de inclusión y formaron parte de este estudio. 


\section{Características de los estudios}

Los estudios seleccionados se publicaron entre 2009 y 2019, con un aumento notable desde 2015 (6/12), lo que refleja que es un área de la educación médica especialmente contingente. La mayor parte de ellos se realizaron en los Países Bajos (4/12), mientras que el resto provino de diferentes países, que incluyen Japón, Estados Unidos de Norteamérica, Canadá, Suecia, Alemania, Australia y Nueva Zelanda. Es posible inferir con esto que el uso de tutores clínicos en la enseñanza de la medicina es una práctica presente en universidades de todo el mundo. No fueron encontrados reportes latinoamericanos, lo que es un reflejo de la baja producción de investigación científica sobre educación médica en Latinoamérica ${ }^{6,7}$. La metodología utilizada en estos estudios fue cualitativa, cuantitativa o mixta. Para recopilar la información, se utilizaron cuestionarios, entrevistas semiestructuradas e instrumentos específicos para evaluar la calidad de los tutores. Un resumen de cada estudio se presenta en la Tabla 2.

\section{Temas comunes encontrados en los estudios}

De los 12 estudios seleccionados, surgieron 106 características, que se agruparon en 46 atributos, que finalmente se clasificaron en 3 dimensiones: genérica, pedagógica y disciplinaria.

La dimensión genérica, que tenía un peso relativo de $46,2 \%$, se dividió a su vez en características personales, actitudinales y comunicativas. La dimensión pedagógica tuvo un peso relativo de $43,3 \%$, y se dividió en estrategias de aprendizaje-enseñanza, planificación-gestión y aptitudes pedagógicas. Finalmente, la dimensión disciplinaria obtuvo un peso relativo de $10,3 \%$. En cada uno de ellos se incluyeron todos los atributos correspondientes mencionados en los artículos, que se presentan en la Tabla 3. A continuación, se realiza una descripción detallada de cada dimensión y los atributos incluidos.

\section{Dimensión genérica}

Corresponde al conjunto de características, actitudes y conocimientos transversales que se requieren en cualquier área profesional y que son transferibles a una gran variedad de campos ${ }^{8}$.

Dentro de la dimensión genérica, las características actitudinales fueron las más mencionadas. El respeto se destaca como un atributo fundamental de las características de actitud, mencionado en 6 de los 12 estudios $^{9-15}$. Las descripciones que se catalogaron dentro del atributo respeto son, por mencionar algunas, tratar a los residentes por igual ${ }^{9}$, respeto por las limitaciones de los estudiantes sin ser peyorativo ${ }^{12} \mathrm{y}$ tratar a los pacientes con respeto $^{13}$. Otro atributo que se considera importante es que los estudiantes pueden identificar en su tutor clínico un "modelo a seguir", mencionado en 5 de 12 estudios $^{10,11,13-16}$, esto es, observar características que vale la pena imitar ${ }^{10,11,13}$, y que representan un ejemplo de desempeño para los estudiantes como futuros médicos ${ }^{13}$. Además, que los tutores clínicos puedan motivar a sus estudiantes ${ }^{11,16} \mathrm{y}$ demostrar compromiso con su trabajo como docentes ${ }^{9,10,14,17}$, son atributos bien evaluados. También, el tutor clínico debe ser entusiasta ${ }^{9,10,13}$, y apoyar a sus estudiantes cuando lo necesiten ${ }^{9,13}$. La humildad ${ }^{9,12,13}$ es otro atributo relevante, es decir, el tutor ideal debe admitir cuando hay algo que no sabe y debe darse cuenta de sus propias limitaciones ${ }^{12,13}$.

Entre las características comunicativas, las más valoradas son que los estudiantes puedan acercarse a sus tutores cuando los necesitan ${ }^{9,11,13}, y$ que los tutores reciban a los estudiantes de manera adecuada $^{9}$. Además, que el tutor clínico propicie $\mathrm{y}$ fomente espacios de consulta y reserve tiempo para sus estudiantes. En general, se espera que el tutor clínico tenga buenas habilidades de comunicación con sus estudiantes y entre grupos ${ }^{18}$, con técnicas de comunicación verbal y no verbal ${ }^{11}$, que permiten al tutor expresar ideas de manera clara y comprensible.

Finalmente, dentro de las características personales, los estudiantes esperan que su tutor tenga sentido del humor ${ }^{11,12}$, tenga una buena apariencia personal $^{11}$, sea interesante ${ }^{9}$, sea carismático ${ }^{19} \mathrm{y}$ tenga rasgos de personalidad adecuados ${ }^{11}$.

\section{Dimensión pedagógica}

Corresponde, por un lado, al conjunto de destrezas y habilidades que se desarrollan y se muestran exclusivamente en el contexto pedagógico del maestro e involucran, principalmente, los métodos utilizados para facilitar el aprendizaje; $y$, por otro lado, hace referencia a las prácticas de planificación y gestión de la enseñanza ${ }^{8,20}$. En el área pedagógica, las estrategias de enseñanza y aprendizaje tuvieron el mayor número de menciones, siendo la retroalimentación la característica más valorada, mencionada en 5 de los 12 estudios $^{9,10,12-14}$. Las descripciones asociadas con la 


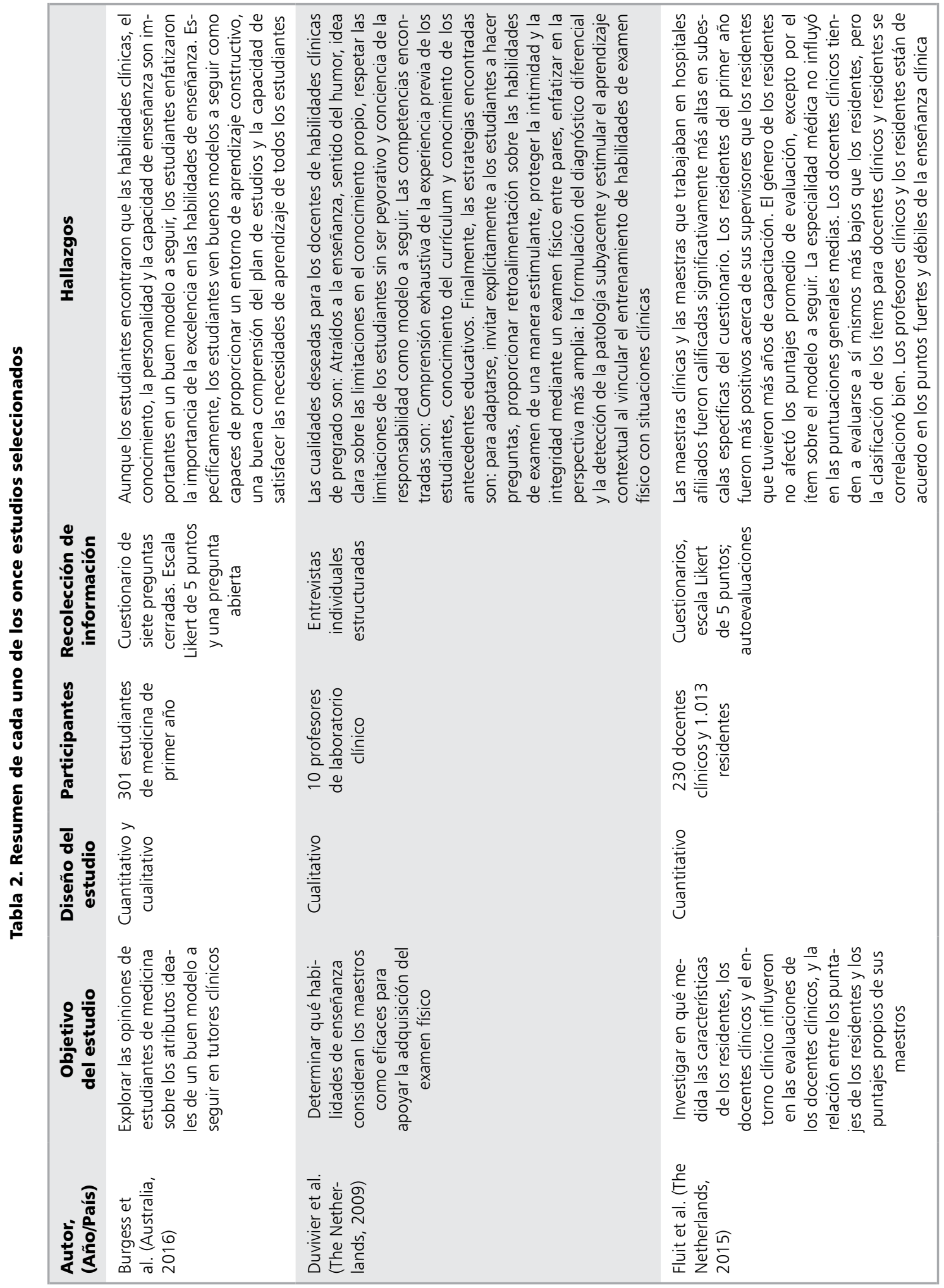




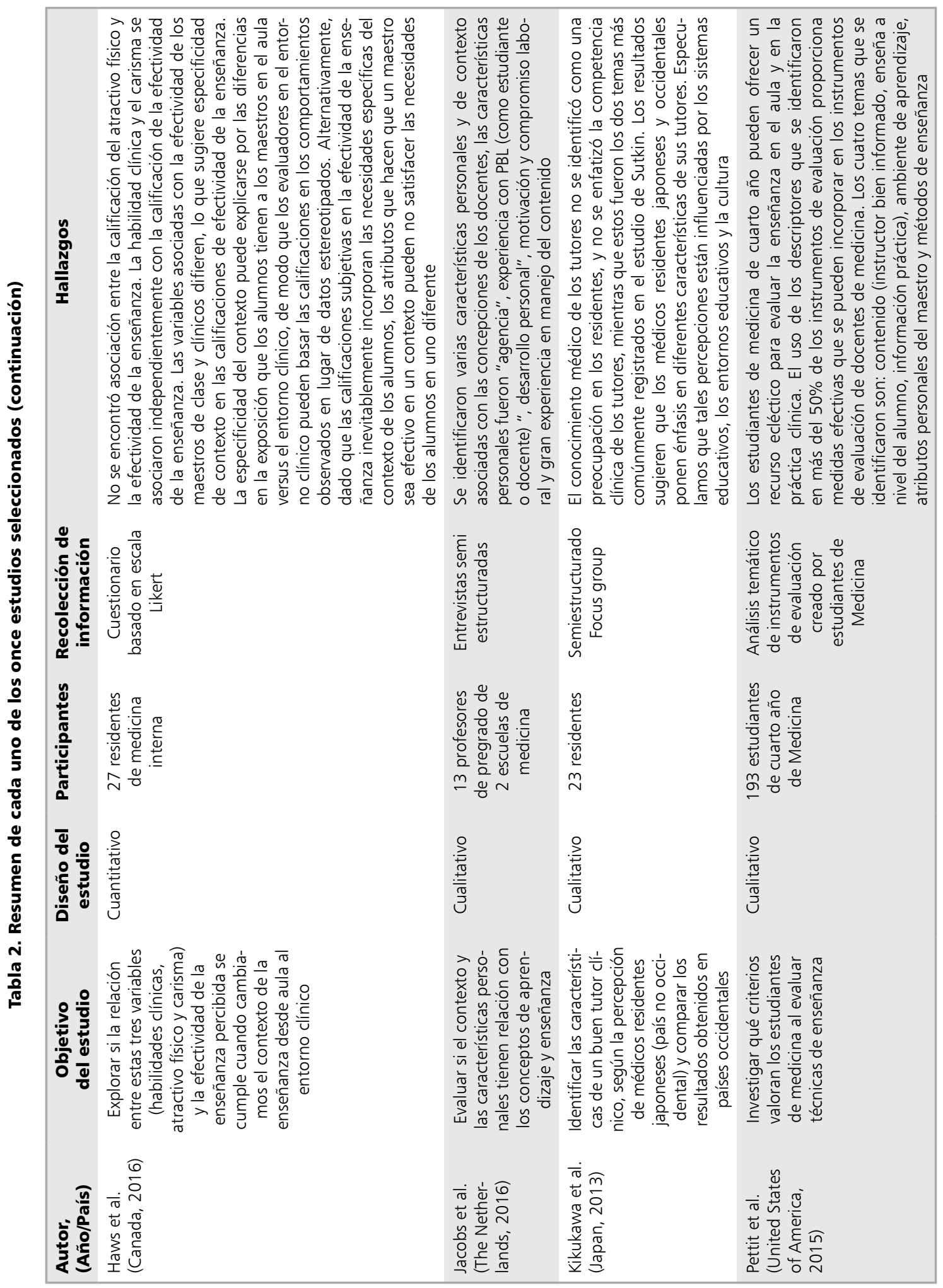




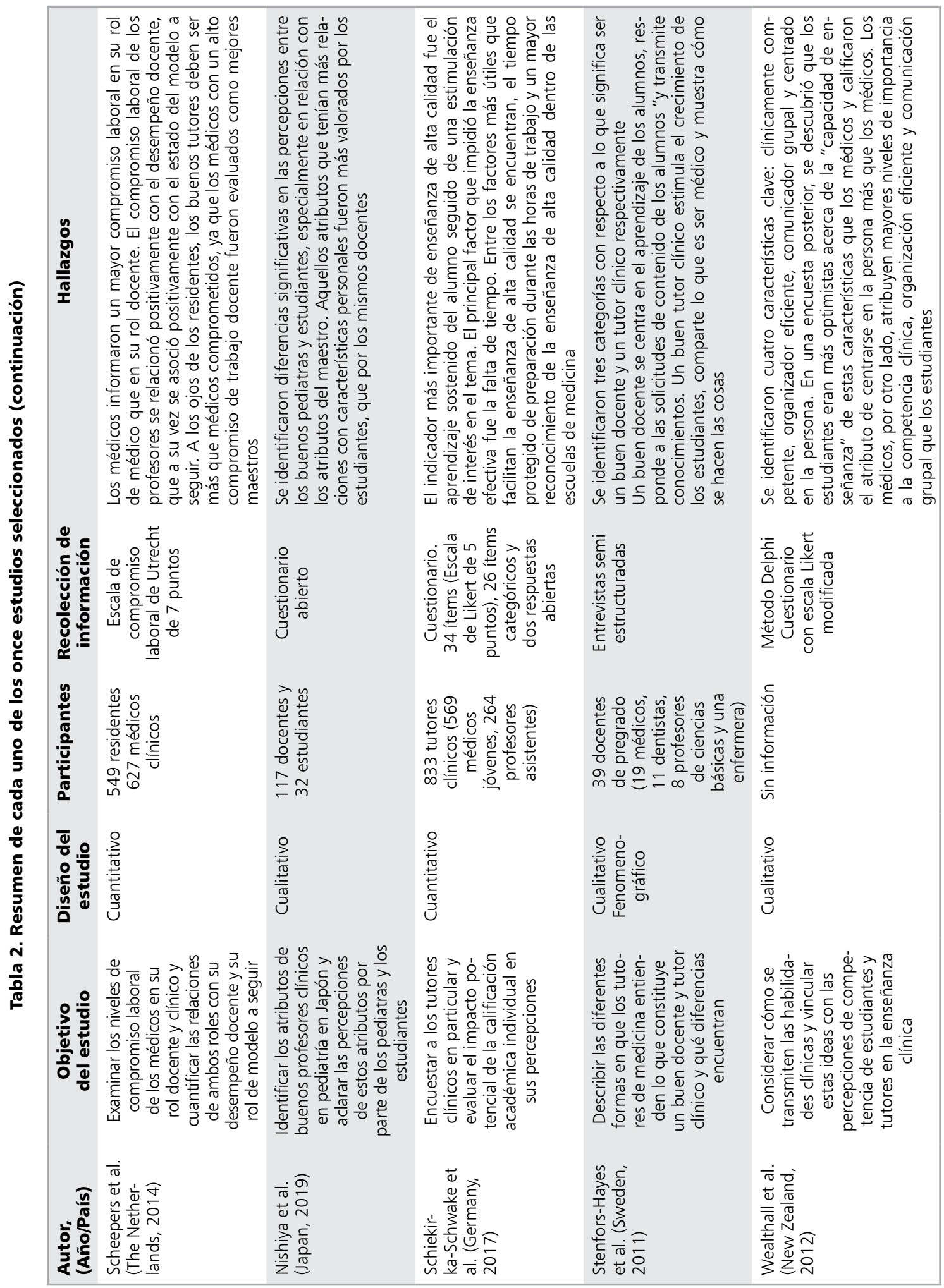


Tabla 3. Atributos de las dimensiones genérica, pedagógica y disciplinaria
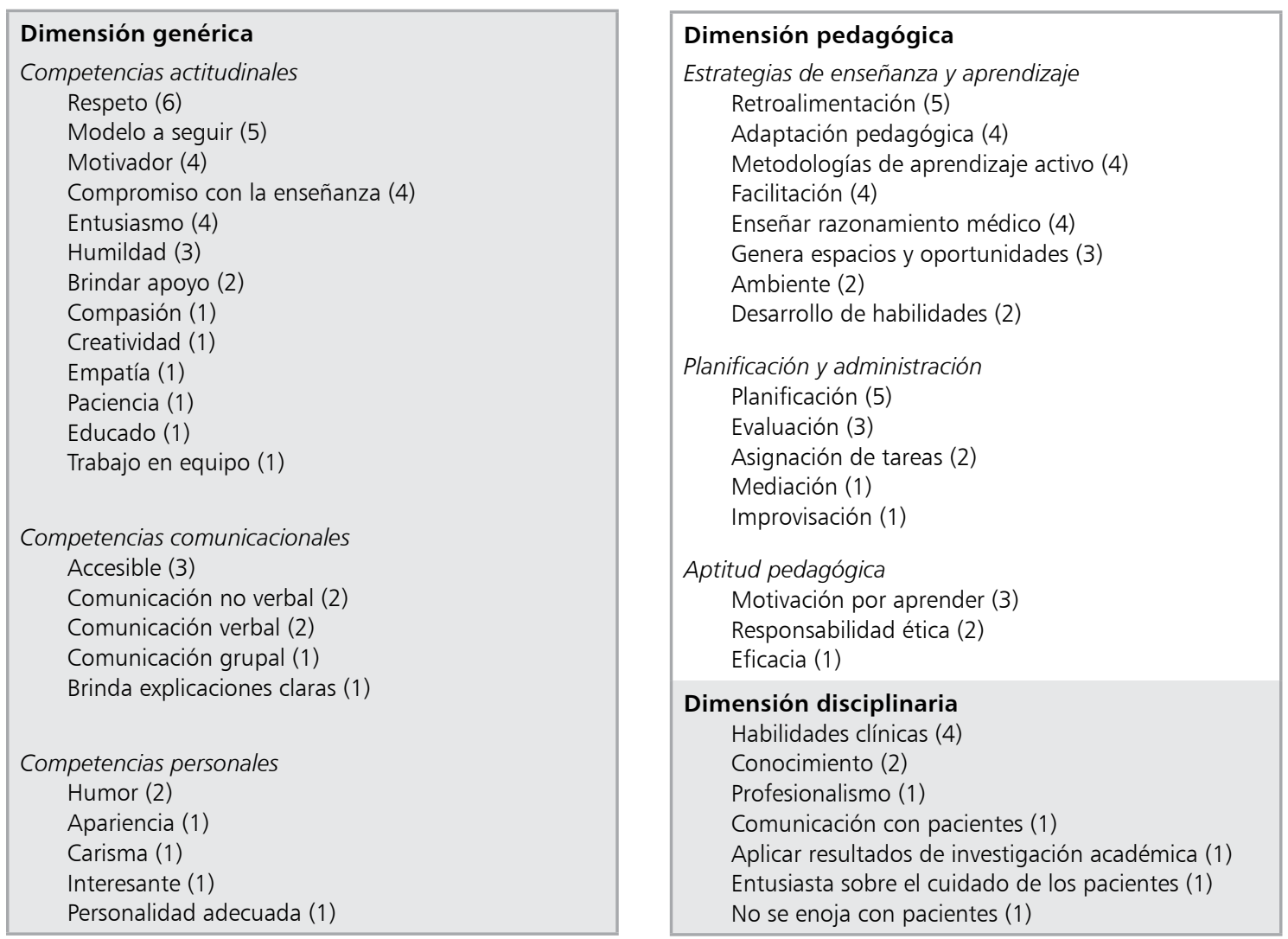

La primera dividida en tres categorías: actitudinal, comunicacional y personal. La segunda dividida en tres categorías: estrategias de enseñanza y aprendizaje; planificación y manejo; y aptitud pedagógica. La tercera sin subcategorías. La frecuencia en que cada atributo fue mencionado en los diferentes artículos se encuentra entre paréntesis al lado de cada atributo.

retroalimentación fueron: proporcionar retroalimentación sobre las habilidades del examen físico de una manera estimulante ${ }^{12}$, hacer observaciones concretas de los estudiantes ${ }^{13}$ y recordarles retroalimentaciones previas de una manera que no sea condescendiente ni insultante ${ }^{13}$. La adaptación pedagógica, entendida como la habilidad del tutor clínico para acomodar el estilo de enseñanza según las necesidades de cada estudiante, fue mencionada en 4 de los 12 estudios ${ }^{10,12,13,18}$. Otras características destacadas con 4 apariciones dentro de la categoría "estrategias de enseñanza y aprendizaje" fueron: metodologías de aprendizaje activo, ${ }^{9,17-19}$ y facilitación ${ }^{9,13,16}$. Esta última entendida como la capacidad de crear situaciones en las cuales el estudiante pueda desarrollar nuevas habilidades por su cuenta ${ }^{9,13}$.

En el área de "planificación y administración”, la planificación aparece como la característica más importante, con 5 menciones ${ }^{10,12-14,18}$.

Finalmente, dentro de la aptitud pedagógica, la motivación por aprender es una característica deseada en los tutores clínicos, de acuerdo con 3 de los 12 estudios ${ }^{12-14}$.

\section{Dimensión disciplinaria}

Corresponde al conocimiento, habilidades y aptitudes del tutor, consideradas como mínimos necesarios para el campo disciplinario que enseña ${ }^{8,21}$. Este grupo fue el que obtuvo la menor cantidad de menciones, y la característica que destacó, con 4 apariciones ${ }^{10,13,19,22}$, fue poseer adecuadas habilidades clínicas, es decir, contar con las destrezas necesarias para el óptimo desempeño en la práctica clínica, y lograr así transmitir esas habilidades a sus estudiantes. 


\section{Discusión}

Se identificaron 46 atributos en los 12 estudios seleccionados, los que pudieron ser clasificados en una de las tres dimensiones propuestas. La distribución de los atributos dentro de las tres dimensiones fue heterogénea, destacando las dimensiones genérica y pedagógica por sobre la disciplinaria.

De acuerdo con esto, los atributos que un tutor clínico puede presentar son muy diversos. Estos atributos cubren distintas áreas, clasificadas en la literatura ${ }^{8}$ y en el presente estudio en dimensiones relacionadas a competencias genéricas, pedagógicas y disciplinares. Todas las dimensiones presentaron al menos un atributo que tenía menciones en tres o más de los estudios seleccionados, equivalente a aproximadamente $25 \%$ de los estudios, valor que hemos designado como el umbral para ser considerado un atributo significativo. Por lo tanto, un tutor clínico de excelencia debe poseer un conjunto de atributos que abarquen las dimensiones genérica, pedagógica y disciplinaria para alcanzar la excelencia.

No obstante, los estudios seleccionados identificaron los atributos del tutor clínico en su gran mayoría desde la perspectiva del estudiante y no del tutor. Sin embargo, no se hace diferencia entre estudiantes de pre y postgrado. Al analizar el detalle de los datos obtenidos, la dimensión genérica obtuvo la mayor aparición en los estudios, concentrando 46,2\% de las menciones, sin embargo, estas se distribuyeron entre 23 atributos diferentes. Se puede observar que hay múltiples atributos que no superan el umbral establecido. Solo hay 7 atributos que exceden este límite: ser respetuoso, ser un modelo a seguir, ser motivador, el compromiso docente, ser entusiasta, ser humilde y ser accesible. Estos serían los más valorados por los estudiantes al evaluar a su tutor clínico. Se infiere que las competencias genéricas son importantes en los tutores clínicos, pero existe una gran dispersión acerca de cuáles serían los atributos fundamentales específicos. Una posible explicación es que este tipo de competencias está condicionado por el contexto, la cultura y las características de cada persona. Cabe destacar las implicaciones de que el tutor clínico se establezca como un modelo a seguir, ya que considera cuestiones éticas y del comportamiento moral, acordes a la formación de buenos profesionales y mejores personas ${ }^{1,23}$.
La dimensión pedagógica tuvo menor mención que la genérica, con $43,3 \%$, sin embargo, existe una mayor concentración de estas, ya que solo se distribuyeron entre 16 atributos. Hay 9 atributos que exceden el umbral: brindar retroalimentación, adaptación pedagógica, metodologías de aprendizaje activo, facilitación, generación de espacios y oportunidades, enseñar razonamiento médico, planificación, evaluación y motivación para aprender. Existe mayor acuerdo entre los estudios sobre cuáles son los atributos pedagógicos que debe tener un tutor clínico de excelencia. Esto puede deberse al hecho de que, a diferencia de las competencias genéricas, las habilidades pedagógicas se identifican mejor como prácticas educativas de excelencia.

La dimensión disciplinaria fue la menos mencionada, con $10,3 \%$. El único atributo que superó el umbral fue poseer habilidades clínicas. Debido a la baja frecuencia de aparición de esta dimensión, pareciera no ser un elemento que los estudios consideran trascendente, tal vez, porque se espera que cada tutor clínico posea el conocimiento necesario de su disciplina para realizar tareas de enseñanza. Aparentemente, para ser un buen tutor clínico, no basta con el dominio de la medicina, sino que es necesario el desarrollo de atributos genéricos y pedagógicos capaces de provocar un aprendizaje significativo en la mayoría de los estudiantes. Es decir, se requiere de una visión integral sobre las características de lo que comprendemos como un buen tutor clínico, y en concordancia con las expectativas de docentes y estudiantes ${ }^{24}$.

En conclusión, los resultados mostraron que las dimensiones genérica y pedagógica fueron aquellas que tuvieron una mayor representación en la literatura, en comparación con la dimensión disciplinaria. Esto podría explicarse en que se supone que la dimensión disciplinaria es la mínima necesaria para poder ejercer como tutor, y no hace una diferencia en cómo se percibe su desempeño. En cambio, las dimensiones genérica y pedagógica marcan la diferencia a la hora de identificar un mejor tutor clínico.

\section{Limitaciones}

Dentro de las limitaciones en la aplicación del protocolo PRISMA se encuentra la imposibilidad de realizar el registro del protocolo de investi- 
gación utilizado en una base de datos pública previo al desarrollo y publicación del trabajo de investigación.

Solo incluimos estudios publicados en español o inglés, por lo que es posible que información relevante publicada en otros idiomas no haya sido incluida. Además, no buscamos en la literatura gris, lo que deja de lado la posible información contenida en estas fuentes.

\section{Referencias}

1. Seoane L, Tompkins LM, De Conciliis A, Boysen PG 2nd. Virtues Education in Medical School: The Foundation for Professional Formation. Ochsner J 2016; 16 (1): 50-5.

2. Sutkin G, Wagner E, Harris I, Schiffer R. What Makes a Good Clinical Teacher in Medicine? A Review of the Literature. Acad Med [Internet] 2008; 83 (5). Disponible en: https://journals.lww.com/academicmedicine/Fulltext/2008/05000/What_Makes_a_Good_Clinical_Teacher_in_Medicine_A.7.aspx.

3. Jochemsen-van der Leeuw HGAR, van Dijk N, Wieringa-de Waard M. Assessment of the clinical trainer as a role model: a Role Model Apperception Tool (RoMAT). Acad Med [Internet] 2014; 89 (4): 671-7. Disponible en: https://pubmed.ncbi.nlm.nih.gov/24556764.

4. Jochemsen-van der Leeuw HGAR, van Dijk N, van Etten-Jamaludin FS, Wieringa-de Waard M. The attributes of the clinical trainer as a role model: a systematic review. Acad Med [Internet] 2013; 88 (1): 26-34. Disponible en: https://doi.org/10.1097/ACM.0b013e318276d070.

5. Liberati A, Altman DG, Tetzlaff J, Mulrow C, Gøtzsche PC, Ioannidis JPA, et al. The PRISMA statement for reporting systematic reviews and meta-analyses of studies that evaluate health care interventions: explanation and elaboration. PLoS Med [Internet] 2009; 6 (7): e1000100-e1000100. Disponible en: https://pubmed. ncbi.nlm.nih.gov/19621070.

6. Morán-Mariños C, Montesinos-Segura R, Taype-Rondan A. Scientific production on medical education in Latin America in Scopus, 2011-2015. Educ Medica 2019; 20: $10-5$.

7. Doja A, Horsley T, Sampson M. Productivity in medical education research: an examination of countries of origin. BMC Med Educ [Internet]. 18 de diciembre de 2014; 14 (1): 243. Disponible en: https://bmcmededuc. biomedcentral.com/articles/10.1186/s12909-014-0243-8 [citado el 8 de junio de 2020].
8. Jerez Yáñez Ó, Orsini Sánchez C, Hasbún Held B. Atributos de una docencia de calidad en la educación superior: una revisión sistemática. Estud pedagógicos [Internet]. 2016; 42 (3): 483-506. Disponible en: http://www.scielo.cl/scielo.php?script=sci_arttext\&pi$\mathrm{d}=$ S0718-07052016000400026\&lng=en\&nrm=iso\&tln$\mathrm{g}=$ en [citado el 8 de junio de 2020].

9. Kikukawa M, Nabeta H, Ono M, Emura S, Oda Y, Koizumi S, et al. The characteristics of a good clinical teacher as perceived by resident physicians in Japan: A qualitative study. BMC Med Educ [Internet] 2013; 13 (1): 100. Disponible en: https://bmcmededuc.biomedcentral.com/articles/10.1186/1472-6920-13-100 [citado el 8 de junio de 2020].

10. Burgess A, Oates K, Goulston K. Role modelling in medical education: the importance of teaching skills. Clin Teach [Internet] 2016; 13 (2): 134-7. Disponible en: https://doi.org/10.1111/tct.12397.

11. Pettit JE, Axelson RD, Ferguson KJ, Rosenbaum ME. Assessing effective teaching: what medical students value when developing evaluation instruments. Acad Med 2015; 90 (1): 94-9.

12. Duvivier RJ, van Dalen J, van der Vleuten CPM, Scherpbier AJJA. Teacher perceptions of desired qualities, competencies and strategies for clinical skills teachers. Med Teach 2009; 31 (7): 634-41.

13. Fluit CRMG, Feskens R, Bolhuis S, Grol R, Wensing $\mathrm{M}$, Laan R. Understanding resident ratings of teaching in the workplace: a multi-centre study. Adv Health Sci Educ Theory Pract 2015; 20 (3): 691-707.

14. Scheepers RA, Arah OA, Heineman MJ, Lombarts KMJMH. In the eyes of residents good supervisors need to be more than engaged physicians: the relevance of teacher work engagement in residency training. Adv Health Sci Educ Theory Pract 2015; 20 (2): 441-55.

15. Nishiya K, Sekiguchi S, Yoshimura H, Takamura A, Wada H, Konishi E, et al. Good clinical teachers in pediatrics: The perspective of pediatricians in Japan. Pediatr Int [Internet] 2020; 62 (5): 549-55. Disponible en: https://doi.org/10.1111/ped.14125.

16. Stenfors-Hayes T, Hult H, Dahlgren LO. What does it mean to be a good teacher and clinical supervisor in medical education? Adv Heal Sci Educ [Internet]. 2011; 16 (2): 197-210. Disponible en: https://doi.org/10.1007/ s10459-010-9255-2.

17. Jacobs JCG, van Luijk SJ, van der Vleuten CPM, Kusurkar RA, Croiset G, Scheele F. Teachers' conceptions of learning and teaching in student-centred medical curricula: the impact of context and personal characteristics. BMC Med Educ [Internet]. 2016; 16 (1): 244. Disponible en: https://doi.org/10.1186/s12909-016-0767-1. 
18. Schiekirka-Schwake S, Anders S, von Steinbüchel N, Becker JC, Raupach T. Facilitators of high-quality teaching in medical school: findings from a nation-wide survey among clinical teachers. BMC Med Educ [Internet]. 2017; 17 (1): 178. Disponible en: https://doi. org/10.1186/s12909-017-1000-6.

19. Wealthall S, Henning M. What makes a competent clinical teacher? Can Med Educ J [Internet]. 2012; 3 (2): e141-5. Disponible en: https://europepmc.org/articles/ PMC4563632.

20. Monereo C, Domínguez C. La identidad docente de los profesores universitarios competentes. Educ XX1. 16 de mayo de 2014; 17 (2): 83-104.

21. Carrillo MF, Henríquez SS, Manzi EF. Concepciones de los estudiantes para profesor de matemáticas sobre las competencias profesionales implicadas en la enseñanza de la estadística. Rev Electron Investig Educ. 2011; 13 (2): 113-31.

22. Haws J, Rannelli L, Schaefer JP, Zarnke K, Coderre S, Ravani $\mathrm{P}$, et al. The attributes of an effective teacher differ between the classroom and the clinical setting. Adv Heal Sci Educ [Internet]. 2016; 21 (4): 833-40. Disponible en: https://doi.org/10.1007/s10459-0169669-6.

23. Bain LE. Revisiting the need for virtue in medical practice: A reflection upon the teaching of Edmund Pellegrino. Vol. 13, Philosophy, Ethics, and Humanities in Medicine. BioMed Central Ltd.; 2018.

24. Ilic D, Harding J, Allan C, Diug B. What are the attributes of a good health educator? Int J Med Educ. 2016; 7. 